\title{
Characteristics of a well-functioning chronic pain team: A systematic review
}

\author{
Heather Griffin MHealsc \\ Team Leader Physiotherapy Outpatients, Tauranga Hospital, Tauranga, New Zealand
}

E Jean C Hay-Smith PhD

Rehabilitation Teaching and Research Unit, Department of Medicine, University of Otago, Wellington, New Zealand

\section{ABSTRACT}

Chronic pain is prevalent, affecting $20 \%$ of New Zealanders. The International Association for the Study of Pain recommends that the treatment of chronic pain is provided by a multi-disciplinary team. To investigate the features supporting the development and maintenance of a well-functioning team, this mixed-method systematic review synthesised empirical research of clinician-reported experience of working in chronic pain teams that provide treatment to adults with chronic, non-cancer related pain. After a search of five electronic database, in which 21 studies were retrieved for full-text screening, seven studies were included in the final review. Studies were rated as unclear, satisfactory or good for both quality (the Mixed Method Appraisal Tool) and transferability. Extracted data were thematically analysed; themes had contributions from more than one key paper (i.e. good quality and good transferability). Four features of a well-functioning team appeared most strongly in the data: team philosophy, co-location, stable workforce and communication. These features are congruent with the broader literature on the characteristics of effective health teams in other patient populations and settings. They might also be features that are particularly important for the development and maintenance of effective chronic pain teams that work in the types of teams and settings typical of the New Zealand context.

Griffin, H., \& Hay-Smith, E. J. C., (2019). Characteristics of a well-functioning chronic pain team: A systematic review. New Zealand Journal of Physiotherapy, 47(1), 7-17. https://doi.org/10.15619/NZJP/47.1.02

Key Words: Multidisciplinary, Interdisciplinary, Teamwork, Chronic Pain, Systematic Review

\section{INTRODUCTION}

In 1990, the International Association for the Study of Pain (IASP) recommended chronic pain services be provided by interor multi-disciplinary teams because chronic pain is a complex problem best managed collaboratively (Gatchel, McGeary, McGeary, \& Lippe, 2014; Main, Sullivan, \& Watson, 2008; Turk et al., 2010). New Zealand has nine multi-disciplinary chronic pain centres within the public health system. These specialist pain management services provide care for the approximate $20 \%$ of New Zealanders with chronic, non-cancer related pain (Ministry of Health, 2017). Given the high prevalence of chronic pain and limited resources, these pain centres need to work efficiently to meet service demands and offer integrated and effective pain management. Studies of clinician experiences working in pain teams and centres may offer insights into how teams and teamwork contribute to a well-functioning team.

Several authors have researched and synthesised research on the key features of multi-disciplinary healthcare teams and teamwork (Mickan \& Rodger, 2000; Nancarrow et al., 2013; Xyrichis \& Ream, 2008). Some common features characterising good teams include clear leadership, clarity of vision,

collaboration, a culture of trust and common purpose (Hewitt, Sims, \& Harris, 2014; Nancarrow et al., 2013). Commentators in the chronic pain literature have observed that similar characteristics are features of a well-functioning pain team, such as interdependence, respect, open communication, collaboration and common goals (Turk et al., 2010).
The existing syntheses of the effectiveness and attributes of teamwork in healthcare cover the general patient populations or specific - but not pain-specific - patient populations (Johansson, Eklund, \& Gosman-Hedström, 2010; Korner et al., 2016; Zwarenstein, Goldman, \& Reeves, 2009). Although teamwork within multi-disciplinary chronic pain is seen as necessary to achieve good outcomes (Turk et al., 2010), we have not found any published synthesis of the literature on multi-disciplinary teamwork in chronic pain services. This review aims to describe the features of multi-disciplinary teams and teamwork that aid the development and maintenance of a wellfunctioning chronic pain team.

In common with other studies of teams and teamwork, this review used the systems theory as a framework for examining the existing literature for features supporting the development and maintenance of an effective chronic pain team. A "system" is defined as "a set of interrelated parts that function as a whole to achieve a common purpose" (Samson, Catley, Cathro, \& Daft, 2012, p. 65). This definition could also describe a multidisciplinary healthcare team (Korner et al., 2016; Mickan \& Rodger, 2000; Xyrichis \& Ream, 2008). Using the model in a healthcare team, inputs (e.g. the multi-disciplinary team members, leadership, team culture and learning) and the processes (e.g. collaboration, communication, trust, respect and shared decision-making) bring suggested outcomes (e.g. patient and staff satisfaction, improved treatment outcomes and cost control). 


\section{METHODS}

This was a mixed method systematic review with thematic synthesis. Reporting of the study methods is guided by the "Enhancing transparency in reporting the synthesis of qualitative research: ENTREQ" statement (Tong, Flemming, McInnes, Oliver, \& Craig, 2012).

\section{Inclusion/exclusion criteria}

Eligible studies were published in English and used either a qualitative or quantitative approach to investigate features or processes of multi-disciplinary teams or team work where the team worked with adults (over the age of 21 years) with chronic pain in a public or private chronic pain setting or institution. Studies were excluded if they were opinion pieces, editorials or reviews; or reported research published before 1990 chronic pain teams were not multi-disciplinary before the IASP first recommended this in 1990. Also excluded were studies investigating teamwork where all the members were from the same profession or discipline (e.g. all medical), or the study took place in acute pain settings (i.e. pain duration of less than six months), paediatric or cancer-related pain services.

Data sources and search strategy

Five electronic databases (MEDLINE, EMBASE, PsycINFO, Scopus and (INAHL) from 1990 to 1 April, 2017 were searched. In consultation with an information specialist, and after scoping searches demonstrated that subject headings did not perform well, the final search string used only keywords with slight variations per database. Each database search was refined to balance sensitivity and specificity until each separate database search gave about 150 search records; this was potentially a total of 750 records if none were duplicates. Using the Boolean operator "AND", keywords for "teamwork" and "chronic pain" were combined with two limits (human and English). The Medline search string is in Appendix 1, and other search strings are available from the corresponding author. The reference lists of included studies were hand searched for potentially eligible studies.

\section{Screening}

Search records were uploaded into a reference management system (Endnote X7, Clarivate Analytics), and titles and abstract (if available) were independently screened by two researchers using a checklist based on the inclusion and exclusion criteria. Full text for each potentially eligible paper was retrieved and independently re-screened for a final decision on inclusion. Any disagreements in eligibility were resolved through discussion. Two "elaborations" of the original inclusion criteria were needed, namely that $75 \%$ of the patients being cared for by the team must have had chronic pain, and that the team features and processes being investigated were within the health professional team members, not between team members and the patient.

\section{Data extraction}

Data describing each study (i.e. study aim, design, setting, study participants and team type) were extracted by one researcher (HG) onto a standardised Microsoft Word template and crosschecked by a second researcher (JH-S). A second Microsoft Word template was used to record the extracted findings and interpretation of each study.
Appraisal items and process

The included studies were assessed for bias using the Mixed Methods Appraisal Tool (Pluye et al., 2011). All studies were appraised (HG) and cross-checked (JH-S), with any disagreement resolved through discussion. A quality rating was assigned to each study using the following criteria:

- Unclear: The study's aim had limited relevance to team attributes or processes; AND/OR there was some doubt that the study's purpose, methodology and methods were congruent; AND/OR there were some concerns regarding the trustworthiness of the study.

- Satisfactory: The study's aim had some relevance to team attributes or processes; AND the study's purpose, methodology and methods were congruent; AND the study was considered trustworthy.

- Good: The study's aim directly addressed team attributes or processes; AND the study's purpose, methodology and methods were congruent; AND the study was considered trustworthy.

Studies were also assessed regarding transferability to a context similar to that of a New Zealand chronic pain service based on the type of team, health professionals and pain service described (e.g. not a private, fee-for-service provider). Each study was classified as follows:

- Unclear: The study's authors used the term "multidisciplinary" (or a similar term) to name the type of team under study but did not define the term; AND/OR there was not a clear description of the team studied or the professions involved; AND/OR the context of the team was not described or did not resemble the chronic pain service model in New Zealand; AND/OR the description of the patient population left uncertainty about its composition.

- Satisfactory: The study's authors used the term "multidisciplinary" (or a similar term) to describe the team under study and provided an explanation of the term; AND provided a sufficient description of the professionals involved; BUT the context in which the team worked was not sufficiently described or was atypical of the New Zealand service model for chronic pain; AND/OR the description of the patient population left uncertainty about its composition.

- Good: The study's authors used the term "multi-disciplinary" (or a similar term) to describe the team under study and provided an explanation of the term; AND provided a sufficient description of the professionals involved; AND the context in which the team worked was sufficiently described to suggest it was similar to the New Zealand service model for chronic pain; AND the description of the patient population was clear and did not include an excluded group.

Data synthesis

Initially, data were extracted and tabulated per study. A summary table was then created to summarise the descriptive information (i.e. study aim, design, setting, study participants and team type) for the studies. Extracted findings and interpretation were read and re-read in meaning units - phrase, 
sentence or (sub) paragraph - and then coded using the systems theory model (input, process, outcome) into one or more of three summary tables. These tables reflected inputs (attributes) (e.g. team culture and make-up of staff) or processes (e.g. communication) documented in prior research in teams and teamwork (Hewitt et al., 2014; Mickan \& Rodger, 2000; Nancarrow et al., 2013; Xyrichis \& Ream, 2008). Some meaning units were cross-coded as they described two or more of the following: attribute, process and outcome.

When all data were coded, links between codes were diagrammed to help organise these into themes. Both researchers debated and and came to an agreement on the final themes. In determining the final themes, the "weight" of data in each code was considered. Studies that were of a good quality and had transferability were given greatest "weight" ("key" papers). A theme had data from more than one "key" paper and was supported by data from informative papers. The classification for "unclear", "informative" and "key papers" was:

- Unclear: Unclear in both quality and transferability; OR unclear in either quality or transferability.

- Informative: Satisfactory in both quality and transferability; OR satisfactory in transferability and good in quality.

- Key paper: Good in both quality and transferability; OR good in transferability and satisfactory in quality.

When the themes were agreed, the links in the data between team attributes and processes were diagrammed; meaning, units that were cross-coded were closely examined for such linkages. Diagramming enabled exploration of sequences or other relationships between team attributes and processes as described in each study.

\section{RESULTS}

From 381 records, 21 were retrieved for full text screening, with seven studies included - four qualitative, one quantitative and two mixed methods (Figure 1). Studies were conducted in the United States of America $(n=4)$, Canada $(n=1)$, the United Kingdom $(n=1)$ and Sweden $(n=1)$ (Table 1). Six studies took place in publicly funded healthcare settings, and four of these were undertaken in a hospital outpatient setting with multi-disciplinary teams that resembled those within New Zealand's public health service. A wide variety of disciplines and professions were represented, and some teams included non-registered healthcare workers. However, usually the team members came from registered health professions and were most often physiotherapists, occupational therapists, social workers, nurses, doctors and psychologists.

The study authors named teams as "integrative", "teamlet", "interdisciplinary", "multi-disciplinary", "team based" and "interprofessional". Two studies provided a description of the team type to ensure the "label" was sufficiently understood. However, it was unclear how the other studies differentiated team type. The assessment of quality and transferability (Table 2) resulted in three key, one informative and three unclear studies.

Here, we present four of the seven themes: team philosophy, co-location, stable workforce and communication. These

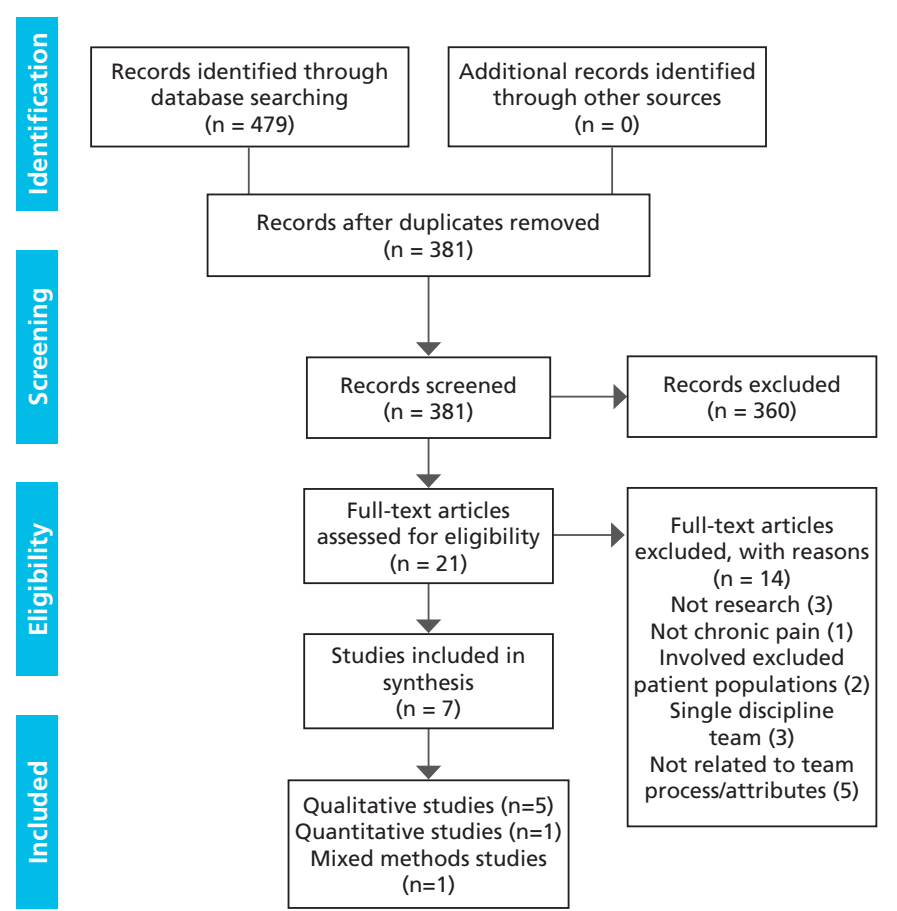

\section{Figure 1: Results of search and eligibility screening}

themes were most strongly supported by data from the key and informative papers. The themes not elucidated here, due to word limitations, are team culture, roles and role boundaries/ blurring. More information about these themes is available from the corresponding author.

\section{Team philosophy}

Well-functioning teams had a specific focus (Cartmill, Soklaridis, \& David Cassidy, 2011), common ground (Hellman, Jensen, Bergström, \& Brämberg, 2016), collective efficacy (Howarth, Warne, \& Haigh, 2012) and shared model of care (O'Connor et al., 2015). Shared philosophy embodies an explicit and collective understanding of the values and purpose of the team; team objectives; or theoretical stance, such as a "broad biopsychosocial framework" (O'Connor et al., 2015).

The shared philosophy was the source of the principles underpinning the team's behaviour and was an important building block for team process, such as coordinated communication, which in turn led to outputs the team considered important, such as consistent messages to patients. However, Hellman et al. (2016, p. 312) found that "having the same basic values did not necessary imply that all members had the same opinions". Thus, a multi-disciplinary team that brings together different professionals who may have different professional cultures, values and models of care has to work together to find a common ground and develop a shared philosophy. Hellman et al. (2016) also found that attention to shared philosophy was a continuing need. For instance, there was a risk of reduced quality of teamwork if shared philosophy was not explicitly discussed with new staff.

\section{Co-location}

The described benefits of sharing team office and treatment space included facilitating access to other team members for discussion and decision-making about clients; an opportunity 
Table 1: Characteristics of included studies

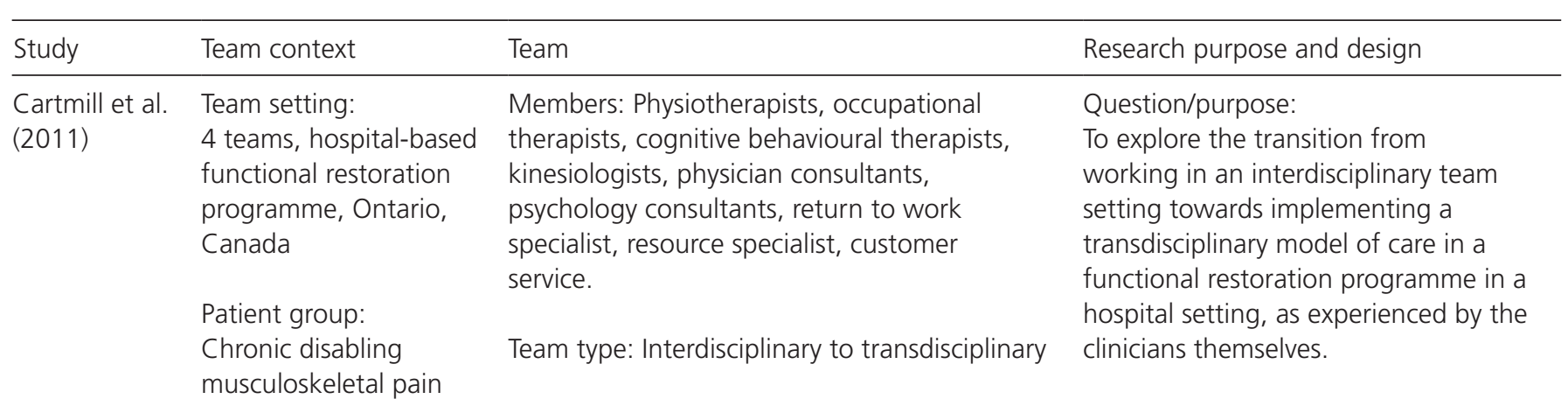

\begin{abstract}
Description/definition:
In an interdisciplinary approach to teamwork, there is a collective identification of client goals that is achieved through co-operation and joint intervention between the various clinicians, the client and his or her family. A transdisciplinary approach incudes the same elements as an interdisciplinary approach, however, each team member becomes so familiar with the roles and responsibilities of other team members that the tasks and functions become, to some extent, interchangeable. By definition, a transdisciplinary team is one in which work across disciplinary boundaries takes place.
\end{abstract}

\section{Members: 11 administrative members and 14 professionals from a variety of disciplines, including medicine, psychology, social work, physical therapy and occupational therapy. \\ Team type: Interdisciplinary \\ Description/definition: No definition given}

Patient group: Chronic pain

Members: Physician, nurse practitioner,

Giannitrapani Team setting:

Primary care practices,

physician assistant supported by

Veterans Affairs Medical

Centres, Los Angeles and Portland, USA

administrative staff. Auxiliary members from pharmacy, social work, mental health and other disciplines.

Patient group: Pain
Team type: Teamlet

Description/definition: 4-member core interprofessional group

\section{Question/purpose:}

To investigate the behavioural effects of a team training model that differentially targeted three categories of group communication and decisionmaking skills.

Design: Observational study - Single subject research design

Question/purpose:

To understand current primary care team-based practices around pain screening, assessment and management.

Design: Qualitative - Inductive 


\begin{tabular}{llll}
\hline Study & Team context & Team & Research purpose and design \\
\hline Haig et al. & Team setting: & Members: Physiotherapists, occupational & Question/purpose: \\
$(2006)$ & University Hospital Spine & therapists, psychologists, social worker/ & This study aims to develop the first \\
& Program & vocational rehabilitation specialist, exercise & codified decision-making process for \\
& USA & physiologist. & individualised treatment planning.
\end{tabular}

Patient group: Chronic Team type: Multi-disciplinary back pain

Hellman et al. Team setting:

(2016) "Rehabilitation warranty"; 9 rehabilitation units 3 Swedish county councils, Sweden

Patient group:

Back pain

Howarth et al. Team setting: 4 pain (2012) management sites in North West England

Patient group: Chronic back pain

\section{O'Connor et Team setting:}

al. (2015) Ambulatory Centre, Academic Hospital, Boston, USA

Patient group: Chronic low back pain
Description/definition: No definition given

Members: Occupational therapists, physicians, psychologists, coordinators, physiotherapists, nurses, social workers and others.

Team type: Team based

Description/definition:

For the concept of team work they cite

Xyrichis and Ream (2008): " . . . a dynamic process involving two or more healthcare professionals with complementary backgrounds and skills, sharing common health goals and exercising concerted physical and mental effort in assessing, planning, or evaluating patient care" (p. 238).

Members: Clinical psychologist, specialist chronic and acute pain nurses, physiotherapists, consultant anaesthetists.

Team type: Interprofessional

Description/definition: Different professionals who share a team identity and who work together in an integrated and interdependent manner, citing Reeves et al. (2010).

Members: Acupuncturist, chiropractor, craniosacral therapist, massage therapist, medical director, movement therapist, occupational therapist, psychiatrist, yoga therapist, Tai chi instructor, nutritionist.

Team type: Integrative

Description/definition: The defining characteristic is that of "a whole person philosophy of patient care" as opposed to interdisciplinary/multi-disciplinary.
Design: Qualitative approach - Case law

Question/purpose: To explore how professionals, without guidelines for implementing interprofessional teamwork, experience the collaboration within team-based rehabilitation for people with back pain and how this collaboration influences their clinical practice.

Design: Mixed methods; Quantitative - cross sectional observational; Qualitative - Inductive content analysis

Question/purpose: To explore person centred care from the perspectives of people with chronic back pain and the interprofessional team who care for them.

Design: Qualitative - Grounded theory

Question/purpose: Multiple qualitative research methods were used to characterise within-team cross-referral and communication amongst jointly trained practitioners representing diverse biomedical and complementary disciplines.

Design: Qualitative - Content analysis

Note: USA, United States of America 
Table 2: Quality assessment of included studies

\begin{tabular}{llll}
\hline Paper & Quality & Transferability & Overall rating \\
\hline Cartmill et al. (2011) & Good & Good & Key \\
Cooley (1994) & Unclear & Unclear & Unclear \\
Giannitrapani et al. (2017) & Satisfactory & Unclear & Unclear \\
Haig et al. (2006) & Unclear & Unclear & Unclear \\
Hellman et al. (2016) & Satisfactory & Good & Key \\
Howarth et al. (2012) & Good & Good & Key \\
O'Connor et al. (2015) & Good & Satisfactory & Informative \\
\hline
\end{tabular}

for congenial relationships to develop; and an opportunity to socialise, collaborate and learn each other's roles and responsibilities (Cartmill et al., 2011; Howarth et al., 2012; O'Connor et al., 2015). Co-location appeared to support the processes that generated more effective teamwork. O'Connor et al. (2015) noted that co-location was a contributor to many informal communication processes, such as hallway conversations, and these were as important for effective teamwork as the formal team meetings and case conferences. When team members did not share the same space, there was less day-to-day contact and reduced opportunity for negotiating roles and developing good interpersonal communication.

\section{Stable workforce}

The longer an individual is in a post, potentially, the more treatment expertise they develop, the more collaborative networks they have and the more familiar they are with the organisational context. Therefore, when multiple team members are in a post for some time, the collective expertise is considerable. The teams under study by Howarth et al. (2012) felt that maturity could take some time: "Although there was no definitive time period in which team maturity evolved, the teams in all four sites described how they had matured over 3 or more years" (p. 494).

Benefits to the team of stable staffing included team intelligence (O'Connor et al., 2015); role familiarity and understanding of individual team members (Howarth et al., 2012); and team maturity (Howarth et al., 2012). Patient-specific outcomes were also thought to benefit when a team was together long enough that a feedback loop was created which enabled the team to see "the consequences of previous decisions" (Haig et al., 2006, p. 1084).

In contrast, staff turnover was reported to drain energy from the team and to slow teamwork down; and dealing with the consequences of staff changes was a "time consuming process" (Hellman et al., 2016, p. 314). Hellman et al. (2016) also found that team members felt they had little time to get to know new team members, to explicitly pass on what they knew as longstanding team members or to rebuild the team. Lack of time for discussion with new team members could lead to a lack of shared values or direction. Hellman et al. (2016) documented the adverse effect of turnover in their quantitative data, stating:
"In total, 30\% reported that staff changes in the past year had influenced their clinical practice, of which $57 \%$ reported that these changes had had negative consequences." (Hellman et al., 2016, p. 311).

\section{Communication}

All seven papers contained data describing communication processes and the importance of these in a well-functioning team. Even within a study, participants "frequently mentioned communication as an important factor in the sustainability of a successful team" (Cartmill et al., 2011, p. 4).

Difficult clinical experiences were usually shared informally, and this included "venting". For instance, "the ability to vent with colleagues was perceived as being particularly important in the FRP (functional restoration programme); it served to maintain a psychologically healthy environment where clinicians felt a continued interest in working within" (Cartmill et al., 2011, p. 4).

Effective communication processes supported learning in the team and good patient care. Fluid information exchange meant knowledge was shared, and this enabled the team members to coordinate their work. For instance, clinicians in the study by Hellman et al. (2016) noted how important this was for giving consistent messages to patients. Genuine dialogue and knowledge exchange provided feedback and support for team members, increased confidence in knowing the roles and skills of others and to comfortably question team decisions.

Three issues with a negative impact for effective communication were noted. First, without a clear process for the "handover" of information from one person to another there is task redundancy and ambiguity (Giannitrapani et al., 2017). Second, time constraints reduced the opportunity for passing assumed knowledge on, especially to "new" people, and prevented indepth discussion (Hellman et al., 2016). Finally, some clinicians experienced "difficulties in getting their voices heard within the team" (Hellman et al., 2016, p. 313); while it was not clear why, the outcome for the clinicians and team was more independent and less collaborative work.

Outcomes of teamwork

Teamwork outcomes were neither the focus of the analysis nor a theme. However, the included studies did make reference to the 
"outputs" of the teamwork described. Teamwork outcomes are the result or product of the input combined with the process. Outcomes of teamwork are thought to encompass benefits to the patient, the staff or the organisation (Borrill et al., 2000; Xyrichis \& Ream, 2008), although actual evidence of patient, staff and organisation benefits of teamwork is known to be weak (Xyrichis \& Ream, 2008; Zwarenstein et al., 2009).

Outcomes for patients included empowering patients to participate in decision-making, (Howarth et al., 2012), receipt of a consistent message (Hellman et al., 2016) and integrated treatment plans ( $\mathrm{O}^{\prime}$ Connor et al., 2015). The organisational benefits included saving time through reducing task redundancy and role ambiguity (Giannitrapani et al., 2017); and that staff rarely resigned and had the ability to cover for each other when they were busy, sick or otherwise absent (Cartmill et al., 2011). Outcomes of effective teamwork for the team were development of a cohesive team, demonstrated in elements such as the power of the team; team intelligence; team dynamic; team credibility; and team collegiality.

\section{DISCUSSION}

This systematic review, which aimed to synthesise the features of teams and teamwork that aid the development and maintenance of chronic pain teams, had findings consistent with the other models and frameworks of "good" interprofessional teamwork in health care. In their realist synthesis on interprofessional healthcare teams, Sims et al. (2015) concluded co-location, length of tenure, face-to-face meetings, role clarity, presence of professional hierarchy, strong leadership, respect and support as the eight most important features influencing teamwork. Furthermore, other systematic reviews of teamwork in other patient populations similarly find key features of effectiveness such as communication, team culture, role clarity (Johansson et al., 2010; Korner et al., 2016), co-location, and team philosophy (Johansson et al., 2010). In summary, it appeared that there was nothing in the limited data currently available specific to chronic pain teams that suggested new or unique features or processes were needed for development and maintenance of a well-functioning chronic pain team.

There were a few features in other systematic reviews which were not evident in the studies that were reviewed. These included leadership (Sims et al., 2015), relevant team members, problem solving and conflict management (Cole, Walter, \& Bruch, 2008; Hewitt et al., 2014; Mickan \& Rodger, 2000; Nancarrow et al., 2013). While these features may be important, there are possibly to few current studies on chronic pain for these attributes to appear strongly.

Incorporating an assessment of quality and transferability as an integral component of the analysis has highlighted a smaller number of features from the larger number mentioned above that may be particularly important in chronic pain settings for development and maintenance of effective teams in New Zealand. These are each considered in more detail below in the context of the broader literature on teamwork.

A stable workforce and team maturity were contributors to an effective interdisciplinary team because high staff turnover had negative consequences for shared philosophy. The importance of a shared philosophy was endorsed by the National Health Service research and subsequent report "The effectiveness of health care teams in the National Health Service" which found that effectiveness is related to clear team objectives (Borrill et al., 2000). Sims et al. (2015) explained that shared purpose is more often visible when absent. Hellman et al. (2016) noted that if a shared philosophy was not explicitly passed on to new members, this could lead to problems for team function, such as a loss of impetus. While Hellman et al. (2016) observed that it was difficult to find time to induct new staff members, it was essential for effective teamwork that they knew and shared the team philosophy.

Co-location or housing of staff together "under one roof" was a particularly strong finding of this review (Cartmill et al., 2011; Howarth et al., 2012; O'Connor et al., 2015) and is supported by the healthcare literature on team work (Mickan \& Rodger, 2000; Molyneux, 2001; Sims et al., 2015). Typically, the shared space was an open plan office, but Cartmill et al. (2011) reported sharing of clinical space too. The informal communication processes and shared learning supported by co-location were a major contributor to congenial relationships and learning about other's roles and responsibilities. Role boundaries were negotiated in ways that led to role clarity as well as deliberate blurring of role boundaries; there were less data about conflict over role boundaries. We hypothesise that co-location offers an opportunity to reduce role conflict, while separate locations may contribute to conflict, especially if non-co-location is also an indicator of a lack of shared line management, which means team members have "split" accountability within the organisation (e.g. to different professional leaders or service managers).

Three key studies in the review highlighted the centrality of "backstage" and informal communication for well-functioning teams (Cartmill et al., 2011; Hellman et al., 2016; Howarth et al., 2012) and how these informal communications were enabled by co-location. "Backstage" communication refers to conversations that are behind the scenes, informal, unstructured and opportunistic in nature; and occur between team members without patients or family present (Lewin \& Reeves, 2011). This type of communication is also reported to be useful in emotional processing, such as when working in difficult situations with patients. For instance, Cartmill et al. (2011) and Hellman et al. (2016) both included data about how an effective team offered a safe environment to "vent" about difficult clinical situations with their colleagues.

In diagramming the relationships between the features identified in our review, we noticed circularity such that a team output - that of team identity - was in turn an input because team identity is part of team culture. It seemed there was a feedforward loop so that the outputs of a well-functioning team feed the inputs and processes of a well-functioning team. Similarly, Mickan and Rodger (2000) in their literature review on the characteristics of an effective team found there was "often a degree of circularity between team structures and processes" (p. 206).

We applied the input-process-output structure from the systems theory of organisations, which is the dominant model used in 
understanding teamwork and known for its simplicity (Borrill et al., 2000; Mickan \& Rodger, 2000). Although the model is reported to be simple, categorising the complex and interactive components of teamwork into three clear-cut components was not. Furthermore, existing models of teamwork also suggest an overlap of some features, for example, different authors have categorised trust and communication as an input or as a process (Borrill et al., 2000; Korner et al., 2016; Xyrichis \& Ream, 2008). Körner et al. (2016) have suggested further research into the use of the input-process-output model to help understand the complex interrelations, and to find effective organisational structures and outcome criteria to assist teamwork.

New Zealander Lennox Thompson (2014) wrote of her experience in chronic pain management and observed the challenges to teamwork as a lack of attention to creating an effective interprofessional team, turf wars over role boundaries, non-co-location of staff, limited induction for new members, lack of agreement on a common approach, lack of a conflict resolution process and inconsistent line-management (e.g. team members reporting to professional leaders rather than a single team leader). These observations of particular local challenges were supported by the review findings, with considerable overlap between Lennox Thompson's (2014) observations and the review findings that shared philosophy, a stable workforce, co-location and good communication were important to the development and maintenance of a well-functioning chronic pain team.

Being deliberately operationalised in the selection criteria for the studies, the findings of this review have been drawn from studies representative of the settings and composition of chronic pain teams in New Zealand. Included studies were mainly conducted in publicly funded health settings, with staff from similar disciplines and a variety of chronic pain patients. One minor difference was the inclusion of social workers in the teams in three studies (Cooley, 1994; Haig et al., 2006; Hellman et al., 2016), which is not common in New Zealand.

The review illustrated an observation made by others that the use of terminology relating to teams is problematic; it seems the terms made from combining the prefixes multi- and interwith "professional" and "discipline" are used inconsistently (Korner et al., 2016; Perrier, Adhihetty, \& Soobiah, 2016). In the chronic pain setting, the inconsistency is unsurprising because, until recently, there was confusion about these terms in the international pain literature. This is reflected in the naming of publicly funded chronic pain services in New Zealand, which are labelled as multi-disciplinary (Northland, Waitemata, Auckland, Counties Manukau, Lakes/Bay of Plenty, Wellington and Dunedin), interdisciplinary (Canterbury) and integrated (Waikato). It is not known whether the type of teamwork differs between these services or whether the variation in naming merely reflects the inconsistencies identified by this review. Until December 2017, the IASP only talked of the multi-disciplinary team (International Association for the Study of Pain, 2014). In December 2017, the IASP announced clear definitions outlining the differences between multi-disciplinary and interdisciplinary care (International Association for the Study of Pain, 2017).
The inconsistency in terminology also made it more difficult to cover all the possible variants in terminology in the search strategy that might describe a chronic pain team comprised of two or more health professions. This difficulty is documented in a bibliometric study by Perrier et al. (2016). Combined with that, this study limited the number of records per database for screening (150 per database) to contain the size of the master's degree project, and excluded studies not published in English. The seven included studies may not be the sum of empirical studies about the features of teamwork in chronic pain teams. Balanced with these limitations was our focus on presenting findings that were found in key studies - those judged trustworthy and useful. With a small evidence base, more studies of the features and process of effective teamwork in chronic pain teams are warranted.

\section{CONCLUSION}

Based on the review findings, chronic pain teams in New Zealand may wish to invest time and energy in the following areas to develop and maintain a well-functioning team:

- Agreeing with and working within a shared philosophy, which includes an induction process for new staff that explicitly communicates team philosophy.

- Offering mutual respect and working non-hierarchically.

- Sharing office space and, ideally, treatment space.

- Team maturity, ideally based on a stable team membership.

- Awareness of others' roles, clarity regarding overlaps in scope of practice, and negotiated role blurring to enable consistent and coordinated care.

- Supporting and seeking out opportunities for informal communication, which includes "venting".

Attention on the development and maintenance of a wellfunctioning team may contribute to improved outcomes for patients, staff and the organisation.

\section{KEY POINTS}

Well-functioning chronic pain teams:

1. Have an agreed and shared philosophy, also known as common purpose or model of care.

2. Share office space.

3. Develop team maturity based on stable staffing.

4. Incorporate formal and informal communication processes, including "venting".

\section{DISCLOSURES}

No funding was obtained for this study. The authors declare no conflicts of interest.

\section{ACKNOWLEDGEMENTS}

We would like to thank Donna Tietjens, Information Specialist, University of Otago, Wellington, for her invaluable assistance in designing and conducting the literature searches for this review. 


\section{ADDRESS FOR CORRESPONDENCE}

Heather Griffin, Physiotherapy Department, Tauranga Hospital, Cameron Road, Private Bag 12024, Tauranga 3143, New Zealand. Telephone: +64 7579 8447. Email: heather. griffin@bopdhb.govt.nz.

\section{REFERENCES}

Borrill, C. S., Carletta, J., Carter, A., Dawson, J. F., Garrod, S., Rees, A., . . West, M. A. (2000). The effectiveness of health care teams in the National Health Service. Birmingham: University of Aston.

Cartmill, C., Soklaridis, S., \& Cassidy, J. D. (2011).

Transdisciplinary teamwork: The experience of clinicians at a functional restoration program. Journal of Occupational Rehabilitation, 21(1), 1-8. https://doi.org/10.1007/s10926-0109247-3

Cole, M. S., Walter, F., \& Bruch, H. (2008). Affective mechanisms linking dysfunctional behavior to performance in work teams: A moderated mediation study. Journal of Applied Psychology, 93(5), 945-958. https://doi.org/10.1037/0021-9010.93.5.945

Cooley, E. (1994). Training an interdisciplinary team in communication and decision-making skills. Small Group Research, 25(1), 5-25. https://doi. org/10.1177/1046496494251002

Gatchel, R. J., McGeary, D. D., McGeary, C. A., \& Lippe, B. (2014). Interdisciplinary chronic pain management: Past, present, and future. American Psychologist, 69(2), 119-130. https://doi.org/10.1037/a0035514

Giannitrapani, K., Ahluwalia S. C., Day, R. T., Pisciotta. M., Dobscha, S. \& Lorenz, K. (2017). Challenges to teaming for pain in primary care. Healthcare. https://doi.org/10.1016/j. hjdsi.2017.06.006

Haig, A. J., Geisser, M. E., Michel, B., Theisen-Goodvich, M., Yamakawa, K., Buchholz, R., ... Sacksteder, J. (2006). The spine team assessment for chronic back pain disability. Part 2: Team decision making and a preliminary outcome study. Disability \& Rehabilitation, 28(17), 1079-1086. https://doi. org/10.1080/09638280600551419

Hellman, T., Jensen, I., Bergström, G., \& Brämberg, E. B. (2016). Essential features influencing collaboration in team-based nonspecific back pain rehabilitation: Findings from a mixed methods study. Journal of Interprofessional Care, 30(3), 309-315. https:// doi.org/10.3109/13561820.2016.1143457

Hewitt, G., Sims, S., \& Harris, R. (2014). Using realist synthesis to understand the mechanisms of interprofessional teamwork in health and social care. Journal of Interprofessional Care, 28(6), 501-506. https://doi.org/10.3109/13561820.2014.939744

Howarth, M., Warne, T., \& Haigh, C. (2012). "Let's stick together" - a grounded theory exploration of interprofessional working used to provide person centered chronic back pain services. Journal of Interprofessional Care, 26(6), 491-496. https://doi.org/10.3109/13561820.2012.711385
International Association for the Study of Pain. (2014). Pain treatment services. Retrieved January 22, 2017, from http:// www.iasp-pain.org/Education/Content.aspx? ItemNumber=1381

International Association for the Study of Pain. (2017). IASP terminology. Retrieved January 22, 2017, from http://www.iasppain.org/Education/Content.aspx? ItemNumber=1698

Johansson, G., Eklund, K., \& Gosman-Hedström, G. (2010). Multidisciplinary team, working with elderly persons living in the community: a systematic literature review. Scandinavian Journal of Occupational Therapy, 17(2), 101-116. https://doi. org/10.3109/11038120902978096

Korner, M., Butof, S., Muller, C., Zimmermann, L., Becker, S., \& Bengel, J. (2016). Interprofessional teamwork and team interventions in chronic care: A systematic review. Journal of Interprofessional Care, 30(1), 15-28. https://doi.org/10.3109/13 561820.2015.1051616

Lennox Thompson, B. (2014, September). Teams in pain management. Pain News, 12(3), 176-179. Retrieved from https://www.britishpainsociety.org/static/uploads/resources/files/ bps_nl_vol12_issue3.pdf

Lewin, S., \& Reeves, S. (2011). Enacting 'team'and 'teamwork': Using Goffman's theory of impression management to illuminate interprofessional practice on hospital wards. Social Science \& Medicine, 72(10), 1595-1602. https://doi.org/10.1016/j. socscimed.2011.03.037

Main, C. J., Sullivan, M. J. L., \& Watson, P. J. (2008). Pain management. Practical applications of the biopsychosocial perspective in clinical and occupational settings (2nd ed.). Edinburgh: Churchill Livingstone.

Mickan, S., \& Rodger, S. (2000). Characteristics of effective teams: a literature review. Australian Health Review, 23(3), $201-$ 208. https://doi.org/10.1071/AH000201.

Ministry of Health. (2017). Annual update of key results 2016/17: New Zealand health survey. Retrieved January 22, 2017 from https://www.health.govt.nz/publication/annualupdate-key-results-2016-17-new-zealand-health-survey

Molyneux, J. (2001). Interprofessional teamworking: What makes teams work well? Journal of Interprofessional Care, 15(1), 29-35. https://doi.org/10.1080/13561820020022855

Nancarrow, S. A., Booth, A., Ariss, S., Smith, T., Enderby, P., \& Roots, A. (2013). Ten principles of good interdisciplinary team work. Human Resources for Health, 11, 19. https://doi. org/10.1186/1478-4491-11-19

O'Connor, B. B., Eisenberg, D. M., Buring, J. E., Liang, C. L., Osypiuk, K., Levy, D. B., \& Wayne, P. M. (2015). Within-team patterns of communication and referral in multimodal treatment of chronic low back pain patients by an integrative care team. Global Advances in Health and Medicine, 4(2), 36-45. https:// doi.org/10.7453/gahmj.2014.076 
Perrier, L., Adhihetty, C., \& Soobiah, C. (2016). Examining semantics in interprofessional research: A bibliometric study. Journal of Interprofessional Care, 30(3), 269-277. https://doi.org $/ 10.3109 / 13561820.2016 .1142430$

Pluye, P., Robert, E., Cargo, M., Bartlett, G., O'Cathain, A., Griffiths, F., ... Rousseau, M. C. (2011). A mixed methods appraisal tool for systematic mixed studies reviews. Retrieved March 6, 2017 from http://mixedmethodsappraisaltoolpublic. pbworks.com

Samson, D., Catley, B., Cathro, V., \& Daft, R. L. (2012). Management in New Zealand. Melbourne, Australia: Cengage Learning.

Sims, S., Hewitt, G., \& Harris, R. (2015). Evidence of a shared purpose, critical reflection, innovation and leadership in interprofessional healthcare teams: A realist synthesis. Journal of Interprofessional Care, 29(3), 209-215. https://doi.org/10.3109/ 13561820.2014 .941459

Tong, A., Flemming, K., Mclnnes, E., Oliver, S., \& Craig, J. (2012). Enhancing transparency in reporting the synthesis of qualitative research: ENTREQ. BMC Medical Research Methodology, 12, 181. https://doi.org/10.1186/1471-2288-12181

Turk, D. C., Stanos, S. P., Palermo, T. M., Paice, J. A., Jamison, R. N., Gordon, D. B., . . Clark, M. C. (2010). Interdisciplinary pain management. Retrieved from http://americanpainsociety.org/ uploads/about/position-statements/interdisciplinary-white-paper. pdf

Xyrichis, A., \& Ream, E. (2008). Teamwork: A concept analysis. Journal of Advanced Nursing, 61(2), 232-241. https://doi. org/10.1111/j.1365-2648.2007.04496.x

Zwarenstein, M., Goldman, J., \& Reeves, S. (2009). Interprofessional collaboration: effects of practice-based interventions on professional practice and healthcare outcomes. The Cochrane Database of Systematic Reviews 3, CD000072, https://doi.org/10.1002/14651858.CD000072.pub2 


\section{Appendix 1}

\section{SEARCH STRATEGY}

Database: MEDLINE (Ovid) 1946 - present

All terms searched as ( ) .mp. , e.g. (team* adj3 conflict)

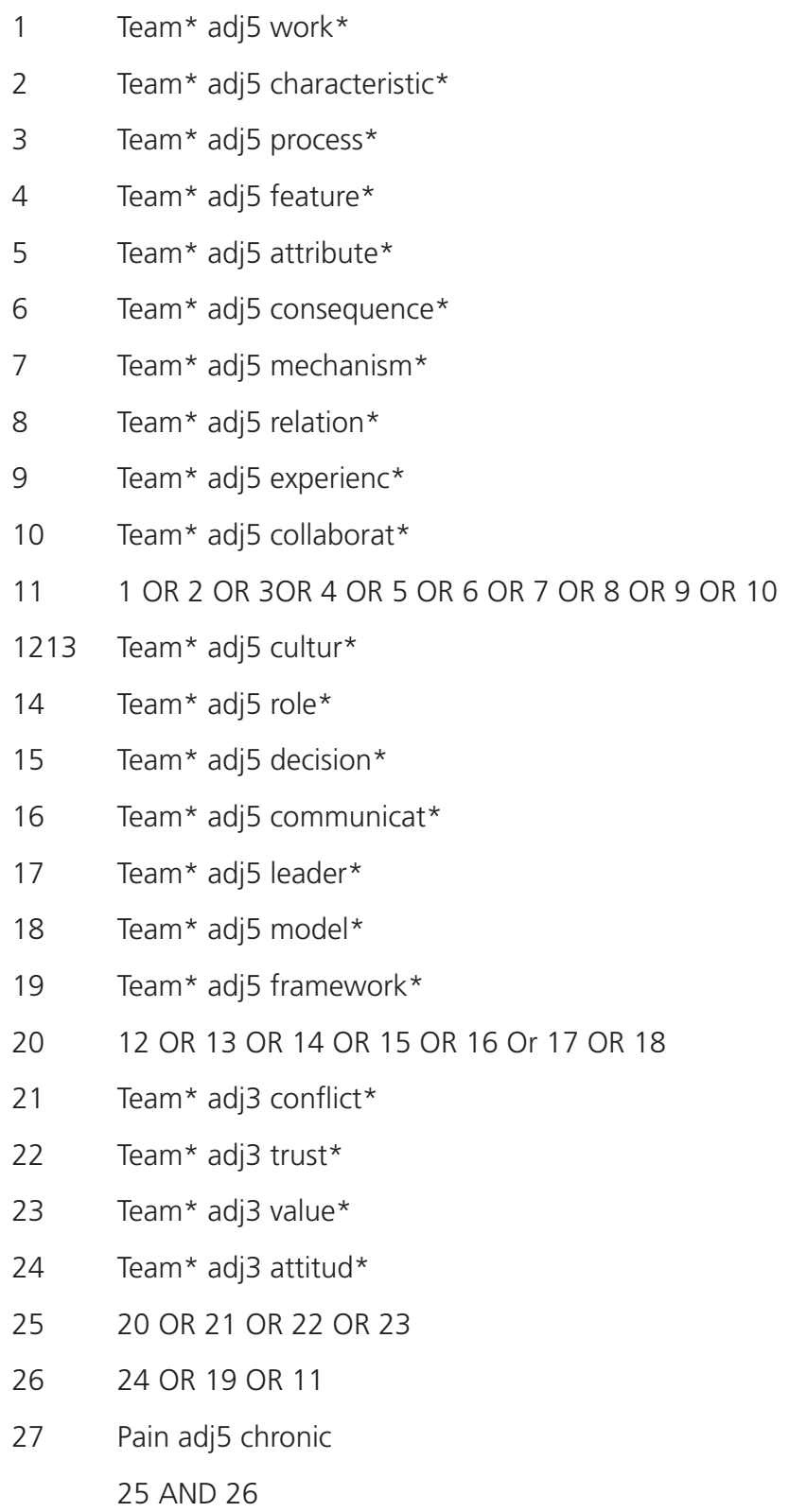

Note: *truncation symbol 